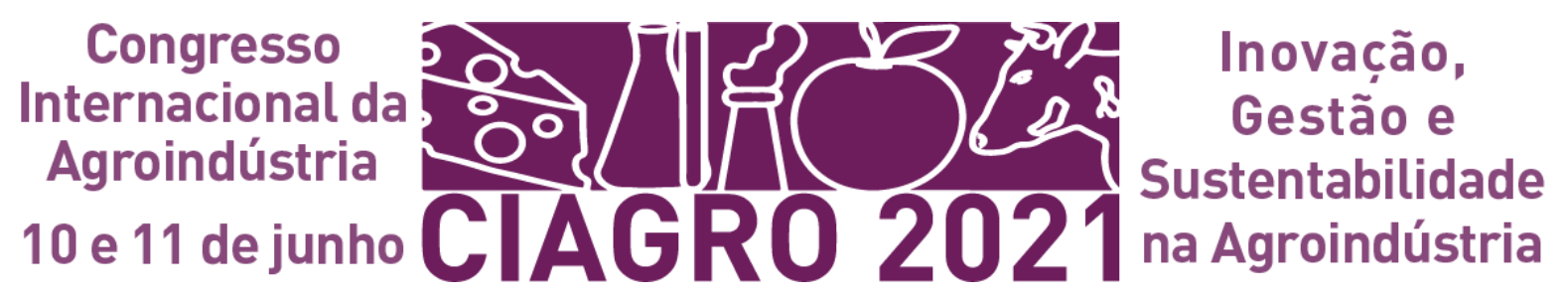

\title{
PRODUÇÃO DE COMPOSTOS FENÓLICOS POR FERMENTAÇÃO EM ESTADO SÓLIDOS COM O USO DE RESÍDUOS AGROINDUSTRIAIS: REVISÃO BIBLIOGRÁFICA
}

\section{PRODUCCIÓN DE COMPUESTOS FENÓLICOS POR FERMENTACIÓN EN ESTADO SÓLIDO CON EL USO DE RESIDUOS AGROINDUSTRIALES: REVISIÓN BIBLIOGRÁFICA}

\author{
PRODUCTION OF PHENOLIC COMPOUNDS BY FERMENTATION IN SOLID \\ STATE WITH THE USE OF AGRO-INDUSTRIAL WASTE: BIBLIOGRAPHIC \\ REVIEW \\ Adryelen Cassiano Martins ${ }^{1}$; Letícia Souza Silva ${ }^{2}$; Juliana Bueno Ruiz ${ }^{3}$; Larine Kupski ${ }^{4}$
}

DOI: https://doi.org/10.31692/IICIAGRO.0118

\begin{abstract}
RESUMO
Os compostos fenólicos são encontrados em diversas matrizes, como nos resíduos agroindustriais. No entanto, podem estar associados a macromoléculas, reduzindo sua disponibilidade. Desta maneira, a fermentação em estado sólido (FES) torna-se uma alternativa promissora para a disponibilização destes compostos em resíduos agroindustriais. O objetivo desta revisão bibliográfica foi identificar na literatura identificar na literatura disponível o uso de resíduos agroindustriais de cereais e leguminosas na fermentação em estado sólido para produção de compostos fenólicos. Como referências de base de dados eletrônicas foram utilizadas as plataformas SCIELO, WEB OF SCIENCE, SCOPUS e SCIENCE DIRECT, analisando estudos publicados nos anos de 2015, 2016, 2017, 2018 e 2020; usando as seguintes palavras chaves: Compostos fenólicos (Phenolic Compounds), Fermentação em estado sólido (Solidstate fermentation), Resíduos agroindustriais (Agro-industrial wastes), Resíduos agroindustriais cereais (Agro-industrial wastes cereals), Resíduos agroindustriais leguminosas (Agro-industrial wastes leguminous), Produção (Production), Síntese Synthesis), flavonóides (Flavonoids), Ácidos fenólicos (Phenolic acids) e Disponibilidade (Availability). Foram selecionados 23 artigos, entre os anos de 2015 a 2020, para avaliar as condições de fermentação mais empregadas para disponibilizacao de compostos fenólicos. Destes estudos 19 referem-se à fermentação de resíduos de cereais e o restante (4), a fermentação de resíduos de leguminosas. O microrganismo mais utilizado foi o fungo filamentoso Aspergillus oryzae. De modo geral, a utilização de resíduos agroindustriais como substratos em fermentação em estado sólido para aumentar a disponibilização de compostos bioativos representa mais uma rota tecnológica em experimentação e que está em grande evolução, o que pode futuramente, diminuir substancialmente os custos de produção, reduzir o acúmulo destes materiais no meio ambiente e agregar valor nestes materiais que antes eram desvalorizados.
\end{abstract}

Palavras-Chave: antioxidante natuais; substratos; fermentação.

\footnotetext{
${ }^{1}$ Graduanda em Engenharia de Alimentos, Universidade Estadual de Maringá, adryelencassiano15@gmail.com

${ }^{2}$ Graduanda em Engenharia de Alimentos, Universidade Estadual de Maringá, lesouzasilva1995@ gmail.com

${ }^{3}$ Doutora em Genética e Melhoramento, Universidade Estadual de Maringá, jbrrebecca2@uem.br

${ }^{4}$ Doutora em Engenharia e Ciências de Alimentos, Universidade Estadual de Maringá, lkupski@uem.br
} 


\title{
RESUMEN
}

Los compuestos fenólicos se encuentran en varias matrices, como en los residuos agroindustriales. Sin embargo, pueden asociarse con macromoléculas, reduciendo su disponibilidad. De esta manera, la fermentación en estado sólido (FES) se convierte en una alternativa prometedora para hacer que estos compuestos estén disponibles en residuos agroindustriales. El propósito de esta revisión de la literatura fue identificar en la literatura para identificar en la literatura disponible el uso de residuos agroindustriales de cereales y legumbres en fermentación en estado sólido para la producción de compuestos fenólicos. Se utilizaron las plataformas SCIELO, WEB OF SCIENCE, SCOPUS y SCIENCE DIRECT como referencias de la base de datos electrónica, analizando los estudios publicados en los años 2015, 2016, 2017, 2018 y 2020; utilizando las siguientes palabras clave: Compuestos fenólicos (Phenolic Compounds), Fermentación en estado sólido (Solid-state fermentation), Residuos agroindustriales (Agro-industrial wastes), Cereales agroindustriales (Agro-industrial wastes cereals), Residuos agroindustriales leguminosos (Agro-industrial wastes leguminous), Producción (Production), Síntesis (Synthesis), flavonoides (Flavonoids), ácidos fenólicos (Phenolic acids) y disponibilidad (Availability). Se seleccionaron 23 artículos, entre los años 2015 a 2020, para evaluar las condiciones de fermentación más utilizadas para la disponibilidad de compuestos fenólicos. De estos estudios 19 se refieren a la fermentación de residuos de cereales y el resto (4), a la fermentación de residuos de leguminosas. El microorganismo más utilizado fue el hongo filamentoso Aspergillus oryzae. En general, el uso de residuos agroindustriales como sustratos en la fermentación en estado sólido para aumentar la disponibilidad de compuestos bioactivos representa una vía más tecnológica en experimentación, que está experimentando una gran evolución, que puede, en el futuro, disminuir sustancialmente los costos de producción, reducir la acumulación de estos materiales en el medio ambiente y agregan valor a estos materiales que antes estaban devaluados.

Palabras Clave: antioxidantes naturales; sustratos; fermentación..

\begin{abstract}
Phenolic compounds are found in several matrices, such as in agro-industrial residues. However, they can be associated with macromolecules, reducing their availability. In this way, solid state fermentation (FES) becomes a promising alternative for making these compounds available in agro-industrial residues. The purpose of this literature review was to identify in the literature to identify in the available literature the use of agro-industrial residues from cereals and legumes in solid state fermentation for the production of phenolic compounds. As electronic database references, the SCIELO, WEB OF SCIENCE, SCOPUS and SCIENCE DIRECT platforms were used, analyzing studies published in the years 2015, 2016, 2017, 2018 and 2020; using the following keywords: Phenolic Compounds, Solidstate fermentation, Agro-industrial wastes, Agro-industrial cereals (Agro-industrial wastes cereals), Leguminous agro-industrial residues industrial wastes leguminous), Production (Production), Synthesis Synthesis), flavonoids (Flavonoids), Phenolic acids (Phenolic acids) and Availability (Availability). Twenty three articles were selected, between the years 2015 to 2020, to evaluate the fermentation conditions most used for making available phenolic compounds. Of these studies, 19 refer to the fermentation of cereal residues and the remainder (4), the fermentation of leguminous residues. The most used microorganism was the filamentous fungus Aspergillus oryzae. In general, the use of agroindustrial residues as substrates in solid state fermentation to increase the availability of bioactive compounds represents yet another technological route under experimentation, which is undergoing great evolution, which may, in the future, substantially decrease production costs, reduce the accumulation of these materials in the environment and add value to these materials that were previously devalued.
\end{abstract}

Keywords: natural antioxidants; substrates; fermentation.

\section{INTRODUÇÃO}

A extensão do território brasileiro possibilita a realização de diversas atividades econômicas, sendo a atividade agrícola a maior responsável pelos valores da balança comercial 
do País. Entretanto, como consequência dessa produção agroindustrial tem-se uma enorme geração de resíduos (Palomino García \& Del Bianchi, 2015b). A exposição direta dos resíduos agroindustriais ao meio ambiente caracteriza uma das principais causas da poluição ambiental, além de significar a perda de material com alto potencial de utilização na produção ou recuperação de diversos compostos com valor comercial (Palomino García \& Del Bianchi, 2015a). Dentre estes compostos destacam-se os compostos fenólicos, que fazem parte de uma das principais classes de antioxidantes naturais. Além de apresentarem atividade antioxidante, tem sido atribuído aos compostos fenólicos benefícios à saúde humana, atuando de maneira a retardar ou prevenir o desenvolvimento de doenças (Palomino García \& Del Bianchi, 2015b).

A fermentação em estado sólido (FES) é uma técnica antiga e vem sendo empregada como alternativa para a produção ou aumento do conteúdo fenólico (Machado et al., 2012). Direcionado pelo contexto atual, no qual se observa uma grande produção de resíduos agroindustriais, utilizá-los como substrato alternativos na fermentação em estado sólido para a produção de compostos antioxidantes é interessante do ponto de vista ambiental e econômico. Desta maneira, o objetivo desta revisão é identificar na literatura disponível o uso de resíduos agroindustriais de cereais e leguminosas na fermentação em estado sólido para produção de compostos fenólicos.

\section{FUNDAMENTAÇÃO TEÓRICA}

Os compostos fenólicos são metabólitos secundários das plantas e são subdivididos em 3 grupos que mais se destacam, como os: ácidos fenólicos, flavonoides e cumarinas (Angelo \& Jorge, 2007). Os ácidos fenólicos por sua vez, são os primeiros na via de síntese, pois são sintetizados a partir da estrutura fenólica mais simples. Estes, possuem um grupo carboxílico ligado ao seu anel aromático que serve como precursor de outros compostos fenólicos mais complexos, como é o caso dos flavonoides (da Silva, 2016). Além disso, os compostos fenólicos podem ser encontrados na sua forma livre, ligados e/ou conjugados e insolúveis (Mira et al., 2008).

Os ácidos fenólicos são subdivididos em duas classes, sendo elas: os ácidos hidroxibenzoicos e os hidroxicinâmicos. Os hidroxibenzoicos são menos abundantes em vegetais de consumo humano, diferente dos hidroxicinâmicos que estão presentes em vários alimentos e bebidas de origem vegetal. Pertencem a este grupo, os ácidos: ácido caféico, $\rho$ cumárico, ferúlico e sinápico que, na maior parte dos alimentos, se encontram esterificados ao ácido quínico, ácido tartárico ou carboidratos e derivados (De Oliveira \& Bastos, 2011).

Em específico, os resíduos proveniente de cereais são ricos em ácidos fenólicos como 
os ácidos ferúlico, vanílico e $p$ - cumárico e flavonoides (Dey et al., 2016). Já os oriundos de leguminosas são ricos em ácidos fenólicos como ferúlico, siringico, cumárico, vanilina, flavonoides, como por exemplo as isoflavonas daidzeína, genisteína e gliciteína (da Silva, Kupski \& Badiale-Furlong, 2018). A diferença encontrada nos resíduos, deve-se ao fato dos compostos fenólicos serem metabólitos secundários de plantas, e sua produção é influenciada por fatores bióticos e abióticos (Furlong et al.,2020). Entretanto, a insolubilidade de compostos fenólicos predominantemente ligados e conjugados reduzem suas biodisponibilidade em cereais e leguminosas. Desta maneira, são estudadas técnicas para melhorar a biodisponibilidade e as propriedades antioxidantes destes compostos, como por exemplo a fermentação em estado sólido (Dey et al., 2016).

A fermentação em estado sólido (FES) trata-se de um processo microbiológico que ocorre na superfície de um sólido - fonte de carbono e energia (Orlandelli et al., 2012), simulando as condições ideais de vida dos fungos filamentosos, que produzem enzimas para degradá-lo, utilizando seus próprios nutrientes para sobreviver. Os fungos são considerados ideais para a FES, pois, se desenvolvem em meios com baixa atividade de água (Ferreira et al., 2011). As variáveis do processo, incluindo pré-tratamento e tamanho de partícula dos substratos, suplementação do meio de crescimento, teor de umidade, concentração do inóculo, temperatura, $\mathrm{pH}$, agitação e aeração, têm um efeito significativo sobre a eficiência dos processos fermentativos em estado solido (Martins et al., 2011) e consequentemente na disponibilização dos compostos bioativos.

\section{METODOLOGIA}

A pesquisa é de abordagem quali e quantitativa e quanto aos procedimentos técnicos utilizou-se de revisão bibliográfica. Como referências de base de dados eletrônicas foram utilizadas as plataformas SCIELO, WEB OF SCIENCE, SCOPUS e SCIENCE DIRECT, analisando estudos publicados nos anos de 2015, 2016, 2017, 2018 e 2020; usando as seguintes palavras chaves: Compostos fenólicos (Phenolic Compounds), Fermentação em estado sólido (Solid-state fermentation), Resíduos agroindustriais (Agro-industrial wastes), Resíduos agroindustriais cereais (Agro-industrial wastes cereals), Resíduos agroindustriais leguminosas (Agro-industrial wastes leguminous), Produção (Production), Síntese Synthesis), flavonóides (Flavonoids), Ácidos fenólicos (Phenolic acids) e Disponibilidade (Availability).

As combinações de busca que foram utilizadas são: "Phenolic acids" AND "Solid state fermentation" AND "Agro industrial wastes" AND "Production OR Availability", "Flavonoids" AND "Solid state fermentation" AND "Agro industrial wastes" AND 
PRINCIPAL, et al.

"Production or availability", "Phenolic compounds" AND "Solid state fermentation", "Synthesis" AND "Phenolic compounds" AND "Solid state fermentation", "Solid state fermentation" AND "Production OR Synthesis OR Availability" AND "Phenolic compounds", "Solid fermentation" AND "Phenolic compounds" AND "Agro industrial wastes cereals OR Agro industrial wastes leguminous" e "Solid state fermentation" AND "Phenolic compounds" AND "Agro industrial wastes". As listas de referências foram exportadas das plataformas eletrônicas e contabilizadas pelo software (EndNote, Thomson Reuters). Foi utilizado o mesmo software para remover as referências duplicadas.

\section{RESULTADOS E DISCUSSÃO}

Como resultado, foram obtidos 23 artigos, 19 estudos referem-se à fermentação de resíduos de cereais (Acosta-Estrada et al.,2019; Călinoiu, Cătoi, \& Vodnar, 2019; De Souza et al.,2019; Janarny \& Gunathilake, 2020; Liu et al., 2016; Maia et al., 2020; Mao et al., 2020; Rashid et al., 2019; Rashid et al., 2015; Razak et al., 2015; Razak et al., 2016; Razak et al., 2017; Razak et al., 2019a; Razak et al., 2019b; Shin et al., 2019; Spaggiari et al., 2020; Tišma et al., 2018; Tu et al., 2020; Yin et al., 2017), e o restante (4), a fermentação de resíduos de leguminosas (Beniwal et al., 2015; Karakurt et al., 2019; Santos et al., 2018; Shi et al.,2020). A quantidade de estudos selecionados para o subprodutos de leguminosas, pode estar associado ao fato de que a maioria dos artigos emprega a fermentação em estado sólido diretamente na leguminosa, sendo excluídos da revisão. Na Figura 1 é possível observar, dentre o conjunto de estudos selecionados, quais são os resíduos agroindustriais de cereais e de leguminosas que mais foram utilizados pelos autores na realização da fermentação em estado sólido como alternativa a produção de compostos fenólicos, ácidos fenólicos e flavonoides. 
Figura 01: Substratos utilizados para produção de compostos fenólicos, ácidos fenólicos e flavonoides na fermentação em estado sólido para as publicações de 2015 a 2020

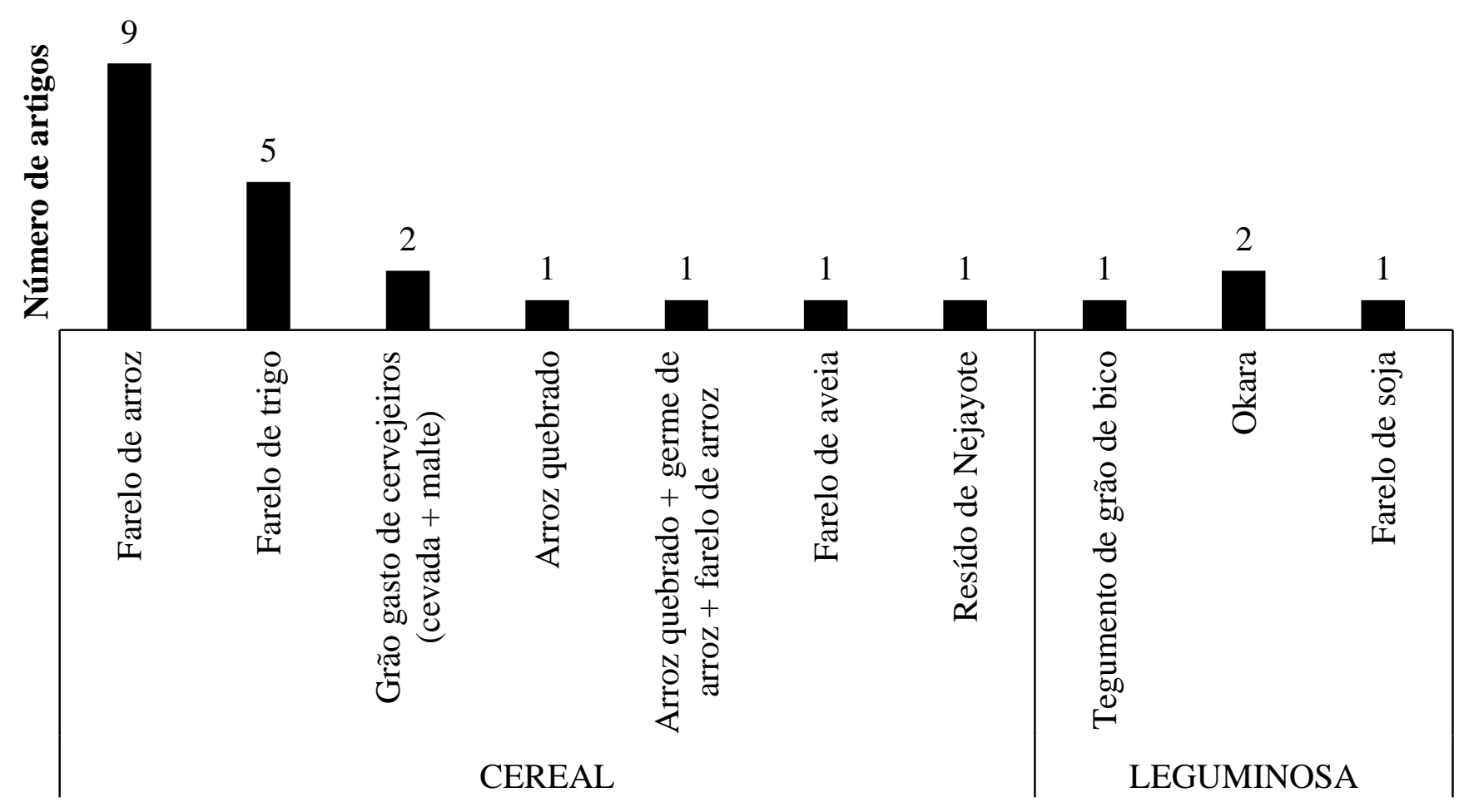

Fonte: Própria (2020).

Para a fermentação em estado sólido de subprodutos de cereais os resíduos que mais se destacaram, foram: o farelo de arroz (N: 9) e farelo de trigo (N:5). Esta escolha justifica-se pelo fato de serem cereais que apresentam alta produtividade, um número considerável de exportação e devido sua composição físico-química, em específico por serem materiais lignocelulósicos (composto por celulose, hemicelulose e lignina).

A degradação microbiana de resíduos agroindustriais está se tornando um caminho mais aplicável para recuperação e produção de compostos bioativos, entre eles, estão os compostos fenólicos e flavonoides. A hidrólise do material lignocelulósico pelas enzimas produzidas pelo fungo durante a fermentação têm grande influência na atividade biológica e biodisponibilidade de compostos bioativos (Mao et al., 2020).

Segundo Statista (2020) estima-se que a produção mundial de arroz na safra de 2019/2020 seja de 495,78 milhões de toneladas métricas e a produção de trigo seja de 764,49 milhões de toneladas métricas. Devido sua alta demanda o processamento industrial destes cereais gera uma grande quantidade de subproduto. O farelo de trigo representa cerca de $15 \%$ 
da massa total do grão, além disso, é constituído por $23,7 \%$ de hemicelulose, $11 \%$ de celulose e 3,6\% de lignina e composto por proteína bruta $(17,4 \%)$, extrato etéreo $(2,1 \%)$, fibra bruta $(7,2 \%)$ e cinzas $(4,2 \%)$ (Kupski et al., 2018). O farelo de arroz representa cerca de 8,5-14,8\% da massa do grão (Walter, Marchezan, \& de Avila, 2008) cerca de 180.000 toneladas métricas de farelo de arroz são geradas por ano (Rashid et al., 2015). É constituído por 2,6\% de celulose, 17,3\% de hemicelulose e 20,9\% de lignina (Lacerda et al., 2010). Além disso, o farelo de arroz é considerado uma boa fonte de nutrientes, rico em fibra alimentar, proteínas, vitaminas e fitoquímicos (Rashid et al., 2015).

Já para a fermentação em estado sólido de leguminosas o resíduo agroindustrial que se mais se destacou foi o Okara, que é um subproduto de soja proveniente do processo de fabricação do leite (tonyu), do queijo (tofu), entre outros produtos relacionados a soja. Assim como o arroz e o trigo, a soja também apresenta alta produtividade e grande parte da sua produção é destinada à exportação. A produção mundial estimada de soja para safra de 2019/2020 é de 336.209 milhões de toneladas métricas (Statista, 2020). Como consequência do aumento do consumo de produtos à base de soja, tem-se uma maior produção de okara.

Segundo Shi et al. (2020), este resíduo é constituído por cerca de 34,7\% de hemicelulose, 43,3\% de celulose e 4,9\% de lignina (Freitas, Natalino, \& Palmiro, 2015) e apresenta alto teor de fibra bruta, proteínas, minerais e compostos fenólicos, além de serem pobres em carboidratos e lipídios,

Considerando que o microrganismo empregado desempenha um grande papel durante a fermentação em estado sólido, na Figura 2 encontra-se a relação dos microrganismos empregados durante os estudos selecionados. 
Figura 02: Microrganismos utilizados para produção de compostos fenólicos, ácidos fenólicos e flavonoides na fermentação em estado sólido para as publicações de 2015 a 2020

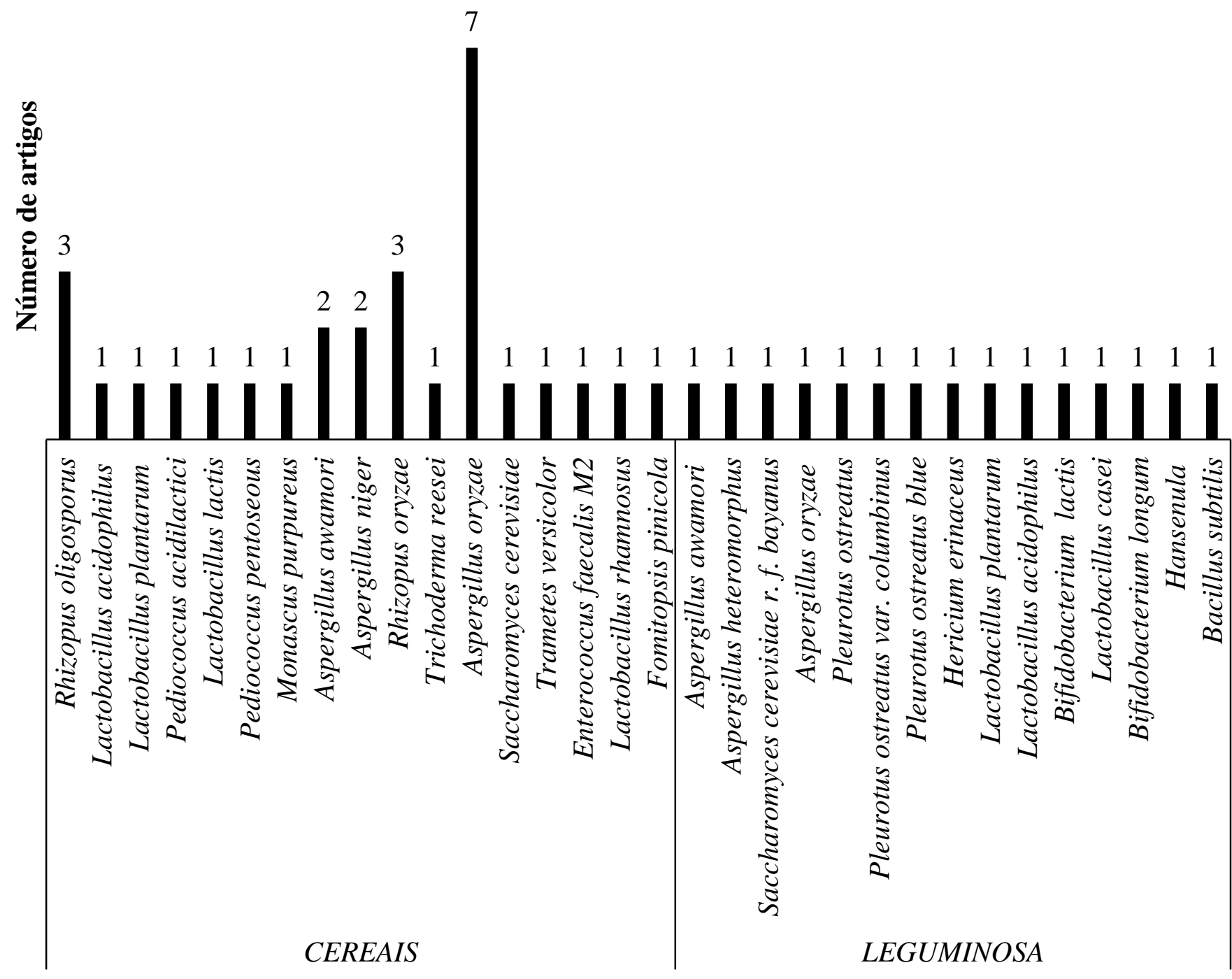

Fonte: Própria (2020).

Dos microrganismos empregados na fermentação de subprodutos provenientes de cereais, os que se destacaram por serem mais utilizados pelos autores, foram: Aspergillus oryzae (N:7), Rhizopus oligosporus (N:3) e Rhizopus oryzae (N:3), diferente da fermentação utilizando subprodutos de leguminosas, no qual nenhum microrganismo foi utilizado em mais de um artigo.

Vale ressaltar, que diversas espécies microbianas estão sendo cultivadas sob fermentação em estado sólido em resíduos agroindustriais, porém os fungos filamentosos, como é o caso de Aspergillus oryzae, Rhizopus oligosporus e Rhizopus oryzae, têm merecido uma atenção especial. Os fungos filamentosos podem se reproduzir tanto de maneira sexuada quanto de maneira assexuada, sendo que a reprodução assexuada ocorre pela fragmentação de suas 
hifas, as quais são responsáveis por colonizar a matriz sólida do substrato. Ambas reproduções (sexuada e assexuada) ocorrem pela formação de esporos, e logo após o fungo filamentoso formar um esporo, o mesmo se separa da célula parental e germina dando origem a novo fungo filamentoso (Pereira, 2016). Portanto, devido suas propriedades fisiológicas, bioquímicas e enzimáticas, os fungos filamentosos conseguem se adaptar em meios com baixa umidade, o que não acontece com as bactérias, consequentemente crescem com mais facilidade sobre substratos sólidos, o que explica a preferência dos autores pelo Aspergillus oryzae, que faz parte do gênero considerado como degradante primário de celulose e lignina (dos Santos et al., 2018).

Por meio da leitura dos estudos selecionados, foi possível identificar na metodologia empregada pelos autores, as análises empregadas e verificar quais foram as mais realizadas para ambos os tipos de substratos (cereal e leguminosa), conforme ilustrado na Figura 3.

Figura 03: Análises empregadas para determinação de compostos fenólicos, ácidos fenólicos e flavonoides na fermentação em estado sólido para as publicações de 2015 a 2020

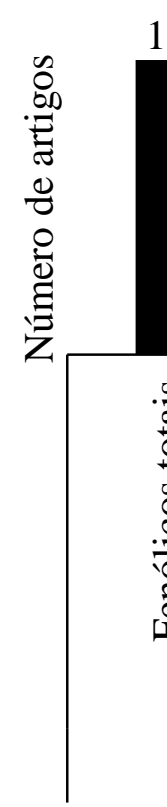

17

15

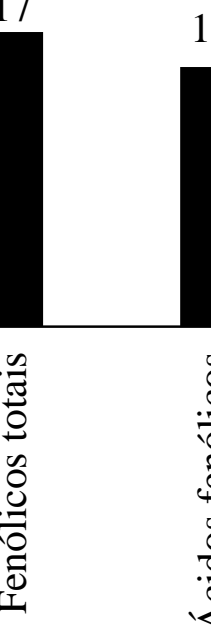

CEREAL
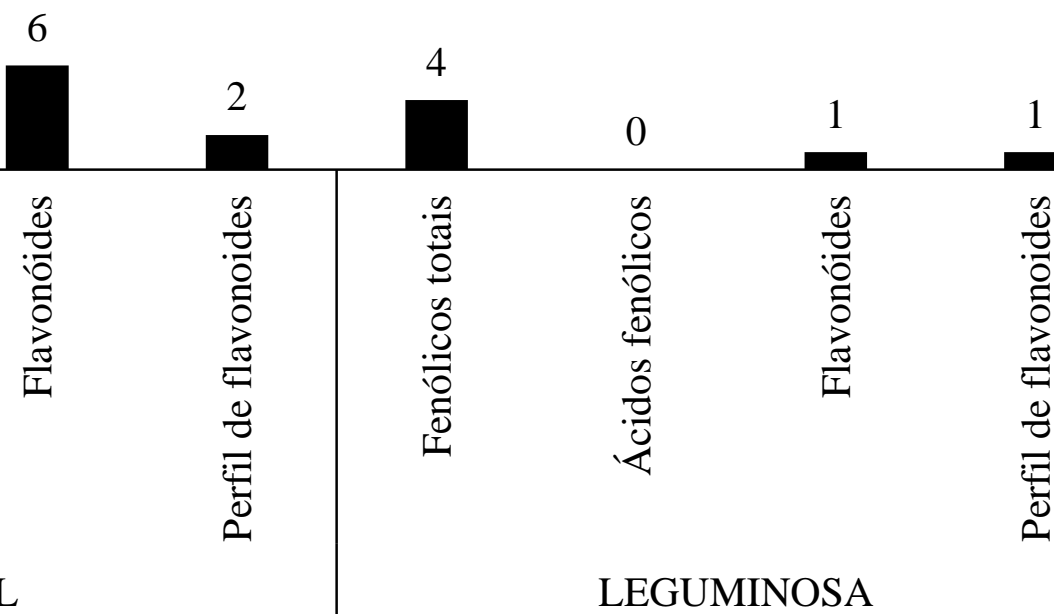

Fonte: Própria (2020).

Nota-se por meio da Figura 3, que a maior parte dos estudos (N: 21) avaliaram a produção e/ou aumento dos fenólicos totais por meio da fermentação em estado sólido. O que se justifica pelo fato de os compostos fenólicos serem metabólitos secundários de plantas, que são subdivididos em três grupos, os: ácidos fenólicos (ácidos benzoicos, cinâmico e seus derivados), os flavonoides (antocianinas, flavonóis e seus derivados) e cumarinas (Angelo \& Jorge, 2007). Portanto, ao determinarem os compostos fenólicos totais estão determinando toda uma classe. No entanto, as pesquisas indicam que a decisão de determinar o perfil de ácidos 
fenólicos e flavonoides está associada ao subproduto escolhido para estudo.

Nos estudos em que se utilizaram subprodutos provenientes de cereais, 17 avaliaram os compostos fenólicos totais, 15 os perfis de ácidos fenólicos, 6 os flavonoides totais e 2 determinaram o perfil de flavonoides. De acordo com Martins et al. (2011), materiais como cereais, vegetais, resíduos de palhas, bagaço, espigas, cascas, etc., são materiais lignocelulósicos, que apresentam em sua fração de lignina numerosos componentes fenólicos, principalmente os ácidos, como os: ferúlico, p- cumárico, seringico, vanílico e p- hidroxibenzoico. Desta maneira, se o subproduto é composto por uma parcela considerável de lignina, portanto a determinação do perfil de ácidos fenólicos pode ser considerada.

Nos estudos em que se empregaram os subprodutos de leguminosas como substrato na fermentação, 4 avaliaram os compostos fenólicos totais e apenas 1 determinou os flavonoides totais e o perfil de flavonoides. Dentre as classes de flavonoides analisadas, as isoflavonas se destacaram, como: Daidzeína, Gliciteínas e a Genisteína. As isoflavonas são encontradas em diversas quantidades em leguminosas e estão presentes, principalmente, como glicosídeos (daidzina e genistina) e agliconas (daidzeína e genisteína) (Angelo \& Jorge, 2007). Os ácidos fenólicos são os primeiros na via de síntese, pois são sintetizados a partir da estrutura fenólica mais simples. Estes ácidos possuem um grupo carboxílico em uma posição do anel aromático que servem como percussores para outros compostos fenólicos mais complexos, como por exemplo os flavonoides (da Silva, 2016).

\section{CONCLUSÕES}

Considerando a revisão realizada neste estudo, a qual considerou como conjunto de amostras diversos estudos em que foi utilizada a fermentação em estado sólido em substratos de cereais e leguminosas para disponibilização de compostos bioativos, foi possível concluir que, poucos estudos foram conduzidos utilizando substratos de leguminosas (N:4), consequentemente a maior parte das amostras foram de estudos conduzidos por meio da utilização de subprodutos de cereais (N:19).

De modo geral, a utilização de resíduos agroindustriais como substratos em fermentação em estado sólido para aumentar a disponibilização de compostos bioativos representa mais uma rota tecnológica em experimentação e que está em grande evolução, o que pode futuramente, diminuir substancialmente os custos de produção, reduzir o acúmulo destes materiais no meio ambiente e agregar valor nestes materiais que antes eram desvalorizados.

\section{REFERÊNCIAS}


Acosta-Estrada, et al. (2019). Effects of solid-state fungi fermentation on phenolic content, antioxidant properties and fiber composition of lime cooked maize by-product (nejayote). Journal of Cereal Science, 90(September), 102837.

Angelo, P. M., \& Jorge, N. (2007). Compostos fenólicos em alimentos - Uma breve revisão. Revista Do Instituto Adolfo Lutz, 66(1), 1-9.

Beniwal, V., et al. (2015). Use of chickpea (Cicer arietinum L.) milling agrowaste for the production of tannase using co-cultures of Aspergillus awamori MTCC 9299 and Aspergillus heteromorphus MTCC 8818. Annals of Microbiology, 65(3), 1277-1286.

Călinoiu, L. F., Cătoi, A. F., \& Vodnar, D. C. (2019). Solid-state yeast fermented wheat and oat bran as a route for delivery of antioxidants. Antioxidants, 8(9).

da Silva, (2016) B. Soja: compostos funcionais e contaminantes. $101 \mathrm{f}$. Dissertação (Mestrado em Engenharia e Ciência de Alimentos) - Universidade Federal do Rio Grande, Programa de pós-graduação em Engenharia e Ciência de Alimentos, Rio Grande.

da Silva, B., Kupski, L., \& Badiale-Furlong, E. Central Composite Design-Desirability Function Approach for Optimum Ultrasound-Assisted Extraction of Daidzein and Genistein from Soybean and Their Antimycotoxigenic Potential. Food Analytical Methods, v. 12, p. 258$270,2018$.

de Souza, T. D., et al. (2019). Profile of phenolic compounds released from rice bran by Rhizopus oryzae and Trichoderma reesei: their relation with hydrolases activity. Journal of Food Science, 84. https: //doi:10.1111/1750-3841.14646

de Oliveira, D. M., \& Bastos, D. H. M. (2011). Biodisponibilidade de Ácidos fenólicos. Quimica Nova, 34(6), 1051-1056. https://doi.org/10.1590/S0100-40422011000600023

Dey, T.B., et al., Antioxidant fenólicos e sua produção microbiana por processo de fermentação submersa e em estado sólido: uma revisão, Trends in Food Science \& Technology (2016), doi: 10.1016 / j.tifs.2016.04.007.

dos Santos, et al. (2018). Fermentação em estado sólido em resíduos agroindustriais para a produção de enzimas: uma revisão sistemática. The Journal of Engineering and Exact Sciences, 04(02), 181-188.

Ferreira, A. N., et al. (2011). Aplicação da fermentação em estado sólido na biotransformação do resíduo do cajá. Revista Acadêmica: Ciência Animal, 9(2), 207.

Freitas, M. M., Natalino, M. T., \& Palmiro, R. A. D. (2015). 55 CBQ - Casca de soja_Extração e caracterização da polpa celulósica e obtenção de carboximetilcelulose. Obtido em: http://www.abq.org.br/cbq/2015/trabalhos/13/8103-

19026.html\#: :text=A\%20composi\%C3\%A7\%C3\%A3o\%20qu\%C3\%ADmica\%20da\%20CS 
,et\%20al.\%2C\%202011). Acessado em 02 de novembro de 2020.

Furlong, E. B., et al. (2020). Use of natural resources from Southern Brazil as a strategy to mitigate fungal contamination. Critical Reviews in Food Science and Nutrition, 1-8.

Janarny, G., \& Gunathilake, K. D. P. P. (2020). Changes in rice bran bioactives, their bioactivity, bioaccessibility and bioavailability with solid-state fermentation by Rhizopus oryzae. Biocatalysis and Agricultural Biotechnology, 23, (December) 101510.

Karakurt, Y., et al. (2019). Chemical, enzymatic, and antioxidant enrichments of full-fat soybean and sunflower meal by Bacillus subtilis (ATCC® 6633TM) fermentation using a solidstate bioreactor. Turkish Journal of Veterinary and Animal Sciences, 43(1), 82-93.

Kupski, L., et al. (2018). Recovery of functional compounds from lignocellulosic material: An innovative enzymatic approach. Food Bioscience, 22(April 2017), 26-31.

Lacerda, D. B. C. L., et al. (2010). Qualidade De Farelos De Arroz Cru, Extrusado E Parboilizado. Pesquisa Agropecuária Tropical, 40(4), 521-530.

Liu, L., et al. (2016). Fermentation and complex enzyme hydrolysis enhance total phenolics and antioxidant activity of aqueous solution from rice bran pretreated by steaming with $\alpha$ amylase. Food Chemistry, 221, 636-643.

Machado, E. M. S., et al. (2012). Growth of fungal strains on coffee industry residues with removal of polyphenolic compounds. Biochemical Engineering Journal, 60, 87-90.

Maia, I. da C., et al. (2020). Effect of solid-state fermentation over the release of phenolic compounds from brewer's spent grain revealed by UPLC-MSE. Lwt, 133(August). https://doi.org/10.1016/j.lwt.2020.110136

Mao, M., et al. (2020). Effect of solid state fermentation by Enterococcus faecalis M2 on antioxidant and nutritional properties of wheat bran. Journal of Cereal Science, 94(April), 102997.

Martins, S. et al. (2011). Bioactive phenolic compounds: production and extraction by solidstate fermentation. A review. Biotechnology advances, 29(3).

Mira, N. V. M. de, et al. (2008). Extração, análise e distribuição dos ácidos fenólicos em genótipos pigmentados e não pigmentados de arroz (Oryza sativa L.). Ciência e Tecnologia de Alimentos, 28(4), 994-1002.

Orlandelli, R., et al. (2012). Enzimas de interesse industrial: produção por fungos e aplicações. SaBios-Revista de Saúde e Biologia, 7(3), 97-109.

Palomino García, L. R., \& Del Bianchi, V. L. (2015a). Capacidade antioxidante em resíduos da indústria cafeeira. Brazilian Journal of Food Technology, 18(4), 307-313.

Palomino García, L. R., \& Del Bianchi, V. L. (2015b). Efeito da fermentação fúngica no teor 
de compostos fenólicos em casca de café robusta. Semina:Ciencias Agrarias, 36(2), 777-786. Pereira, C. B. (2016). Avaliação da produção de enzimas celulolíticas e hemicelulolíticas por fungos isolados do cerrado, costa marinha brasileira e da Antártica, utilizando casca de soja como substrato. 117 f. il., Dissertação (Mestrado em Ciências Farmacêuticas) - Faculdade de Ciências de Saúde, Universidade de Brasília, Brasília.

Rashid, N. Y. A., et al. (2019). Quantification of phenolic compounds changes by Aspergillus oryzae on rice bran fermentation. Food Research, 3(2), 133-137.

Rashid, N. Y. A., et al. (2015). Bioactive compounds and antioxidant activity of rice bran fermented with lactic acid bacteria. Malaysian Journal of Microbiology, 11(Specialissue2), $156-162$.

Razak, D. L. A, et al. (2015). Enhancement of phenolic acid content and antioxidant activity of rice bran fermented with Rhizopus oligosporus and Monascus purpureus. Biocatalysis and Agricultural Biotechnology, 4(1), 33-38.

Razak, D. L. A., et al. (2016). Comparative study of antioxidant activities, cosmeceutical properties and phenolic acids composition of fermented rice bran and coconut testa. Jurnal Teknologi. 2, 29-34.

Razak, D. L. A., et al. (2017). Cosmeceutical potentials and bioactive compounds of rice bran fermented with single and mix culture of Aspergillus oryzae and Rhizopus oryzae. Journal of the Saudi Society of Agricultural Sciences, 16(2), 127-134.

Razak, D. L. A, et al. (2019a). Assessment of fermented broken rice extracts for their potential as functional ingredients in cosmeceutical products. Annals od Agricultural Sciences, 64, 176182.

Razak, D. L. A., et al. (2019b). Brewer's rice-A potential substrate for cosmeceutical bioingredient production by solid state fermentation using Aspergillus oryzae. Malaysian Journal of Microbiology, 15(SpecialIssue4), 260-266.

Santos, V. A. Q, et al. (2018). Solid-state fermentation of soybean okara: Isoflavones biotransformation, antioxidant activity and enhancement of nutritional quality. Lwt, 92, 509515.

Shi, H., et al. (2020). Solid-state fermentation with probiotics and mixed yeast on properties of okara. Food Bioscience, 36(April), 100610.

Shin, H. Y., et al. (2019). Solid-state fermentation of black rice bran with Aspergillus awamori and Aspergillus oryzae: Effects on phenolic acid composition and antioxidant activity of bran extracts. Food Chemistry, 272, 235-241.

Spaggiari, M., et al. (2020). Solid state lactic acid fermentation: A strategy to improve wheat 
bran functionality. Lwt, 118, 108668.

Statista (2020). Grain production worldwide 2019/20. Obtido em: https://www.statista.com/statistics/263977/world-grain-production-by-type/. Acessado em 02 novembro de 2020.

Tišma, M., et al. (2018) Biovalorization of brewers' spent grain for the production of laccase and polyphenols. Journal Institute of Brewing \& Distilling, 124, 182-186.

Tu, J., et al. (2020). Solid state fermentation by Fomitopsis pinicola improves physicochemical and functional properties of wheat bran and the bran-containing products. Food Chemistry, 328, 127046.

Walter, M., Marchezan, E., \& de Avila, L. A. (2008). Arroz: composição e características nutricionais. Ciência Rural, 38(4), 1184-1192.

Yin, Z., et al (2017). Comparison of releasing bound phenolic acids from wheat bran by fermentation of three Aspergillus species. International Journal of Food Science and Technology, 53(5), 1120-1130. 